\title{
Management of invasive Allee species
}

\author{
D. M. Chan ${ }^{\mathrm{a}}$, C. M. Kent ${ }^{\mathrm{a}}$ and D. M. Johnson ${ }^{\mathrm{b}}$ \\ ${ }^{a}$ Department of Mathematics and Applied Mathematics, Virginia Commonwealth University, Richmond, VA, \\ USA; ${ }^{b}$ Department of Biology, Virginia Commonwealth University, Richmond, VA, USA
}

\begin{abstract}
In this study, we use a discrete, two-patch population model of an Allee species to examine different methods in managing invasions. We first analytically examine the model to show the presence of the strong Allee effect, and then we numerically explore the model to test the effectiveness of different management strategies. As expected invasion is facilitated by lower Allee thresholds, greater carrying capacities and greater proportions of dispersers. These effects are interacting, however, and moderated by population growth rate. Using the gypsy moth as an example species, we demonstrate that the effectiveness of different invasion management strategies is contextdependent, combining complementary methods may be preferable, and the preferred strategy may differ geographically. Specifically, we find methods for restricting movement to be more effective in areas of contiguous habitat and high Allee thresholds, where methods involving mating disruptions and raising Allee thresholds are more effective in areas of high habitat fragmentation.
\end{abstract}

\section{ARTICLE HISTORY}

Received 17 August 2016

Accepted 8 May 2017

\section{KEYWORDS}

Allee species; invasion; discrete model; management

\section{Introduction}

The increased international movement of humans and commercial products over the last couple of centuries has facilitated the introduction of unprecedented numbers of nonnative species worldwide (Vitousek, D’Antonio, Loope, \& Westbrooks, 1996). While most of these introductions fail to establish due to environmental unsuitability or stochasticallydriven extinctions, or persist at innocuous population densities, a subset of these introductions becomes economic and ecological pests (Williamson \& Fitter, 1996). A requisite feature of an invasive pest is the ability to expand its range into new territory after introduction, a process that is particularly dependent on trajectories of low density populations at and beyond range edges.

Allee effects, defined as depressed population growth rates at low densities, can be generated by many mechanisms such as the inability to locate mates, a lack of predator satiation, a reduction in social benefits and inbreeding depression; thus, Allee effects are likely common in natural populations (Kramer, Dennis, Liebhold, \& Drake, 2009). Despite the challenges of detecting Allee effects in natural populations (Gregory, Bradshaw, Brook,

CONTACT D.M.Chan dmchan@vcu.edu 
\& Courchamp, 2010), which include inherent difficulties associated with studying low density populations, Allee effects have been detected in a number of species representing a broad diversity of taxonomic groups (Kramer et al., 2009), including invasive species (Anic, Henriquez, Abades, \& Bustamante, 2015; Tobin, Berec, \& Liebhold, 2011). Given that Allee effects are likely widespread and can have large effects on population spread rates, understanding how Allee effects can enhance the efficacy of management practices is important to maximizing return on management effort.

The presence of Allee effects can significantly alter, reduce or prevent species persistence and/or invasion (Johnson, Liebhold, Tobin, \& Bjornstad, 2006b; Keitt, Lewis, \& Holt, 2001; Taylor \& Hastings, 2005). Recent papers have reviewed how management practices can work synergistically or antagonistically with Allee effects in the control of invasive species (Tobin et al., 2011; Suckling, Tobin, McCullough, \& Herns, 2012). Several studies have previously used discrete models involving an Allee effect to investigate population dynamics in local and spatial environments (Chow \& Jang, 2014; Jang, 2006; Johnson et al., 2006b; Li, Song, \& Wang, 2007). In this study we spatially-extended the model from Courchamp (Courchamp, Ludek, \& Gascoigne, 2008), which is a modification of the Ricker model (Ricker, 1954). The model has parameters that capture four types of population processes: negative density-dependent, positive density-dependent and densityindependent effects on population growth and dispersal. Different management strategies act disproportionately on different parameters in this model. We investigate how altering the different population parameters inhibits the expansion of an Allee species. We provide biological context by linking the parameters to common management strategies that are applied to species invasions.

Here, we focus on populations with strong Allee effects, which are defined as having a positive Allee threshold, a density below which the population will decline towards extinction. If this threshold is high then a species may have difficulty persisting and expanding into new territory (Keitt et al., 2001; Lanchier, 2013), particularly if its movement is restricted (Ackleh, Allen, \& Carter, 2007).

In this study, we first justify analytically that the extension of the basic model to a two-patch model exhibits the Allee effect with similar environments. Next we explore how management strategies affect the asymptotic dynamics of the patch populations, specifically whether a management strategy prevents expansion from one patch to another, in landscapes with different characteristics. We show that geographical differences in demographic and landscape features may alter the effectiveness of different management strategies at inhibiting expansion; thus, the most effective strategy may differ across a species' range.

\section{Model and background}

To look at how different management strategies affect the invasiveness of Allee species we start with a modified Ricker model (Ricker, 1954) proposed by Courchamp et al. (2008):

$$
x(t+1)=x(t) \exp \left(r\left[1-\frac{x(t)}{K}\right]\left[1-\frac{A+C}{x(t)+C}\right]\right) .
$$

Here, $x(t)$ is the population density of the $t$ th generation, $r$ is the intrinsic growth rate, $K$ is the carrying capacity, $A$ is the Allee threshold and $C$ is a constant that affects the 
strength of the Allee effect near the Allee threshold. The Allee threshold, which is strictly less than the carrying capacity, is the density where populations larger than this value grow and smaller populations decline. Clearly in this model when the population is below the Allee threshold the exponent is negative giving a negative growth rate, and populations immediately above this threshold have a positive growth rate.

To investigate whether invasion is possible we consider a two-patch model where the species moves from one patch to the other. To create movement between the patches in this closed system, we assume that a percentage of the difference between current patch and the average population between the patches will move from the patch with the higher population to the patch with the lower population. Let $x_{1}(t)$ be the population density in patch 1 and $x_{2}(t)$ be the population density in patch 2 , and define $F: \mathfrak{R}_{+}^{2} \rightarrow \mathfrak{R}_{+}^{2}$ to be

$$
\begin{aligned}
\left(x_{1}(t+1), x_{2}(t+1)\right)= & F\left(x_{1}(t), x_{2}(t)\right) \\
= & \left(F_{1}\left(x_{1}(t), x_{2}(t)\right), F_{2}\left(x_{1}(t), x_{2}(t)\right)\right) \\
= & \left(\left(1-\frac{d}{2}\right) x_{1}(t) \exp \left(r_{1}\left[1-\frac{x_{1}(t)}{K_{1}}\right]\left[1-\frac{A_{1}+C}{x_{1}(t)+C}\right]\right)\right. \\
& +\frac{d}{2} x_{2}(t) \exp \left(r_{2}\left[1-\frac{x_{2}(t)}{K_{2}}\right]\left[1-\frac{A_{2}+C}{x_{2}(t)+C}\right]\right), \\
& \left(1-\frac{d}{2}\right) x_{2}(t) \exp \left(r_{2}\left[1-\frac{x_{2}(t)}{K_{2}}\right]\left[1-\frac{A_{2}+C}{x_{2}(t)+C}\right]\right) \\
& \left.+\frac{d}{2} x_{1}(t) \exp \left(r_{1}\left[1-\frac{x_{1}(t)}{K_{1}}\right]\left[1-\frac{A_{1}+C}{x_{1}(t)+C}\right]\right)\right)
\end{aligned}
$$

where $d \in[0,1]$ is the diffusion rate, and $\mathfrak{R}_{+}^{2}=\{(x, y) \mid x, y \geq 0\}$. Each of the previous parameters in (1) have counterparts in each patch. Here, we assume that growth occurs and then dispersal. $\mathrm{C}$ is assumed to be a species-specific parameter that is not dependent on the patch, whereas depending on the resources within the patch $r, A$ and $K$ may vary.

To determine the possibility of invasion we consider the case where the population is at carrying capacity in patch 1 and is not present in patch 2 . We examine numerically under what parameter sets patch 2 will be successfully colonized, when patch 1 will go extinct, and the resulting non-transient population densities. We consider how expansion of an Allee species from patch 1 into patch 2 is affected by altering three demographic parameters: dispersal, Allee threshold and carrying capacity. We also compare the effectiveness of altering these demographic parameters in species across a range of intrinsic population growth rates, $r$.

Diffusion rate, $d$, can be reduced by restricting the proportion of individuals moving from patch 1 to patch 2 . The number of dispersers can be particularly critical in Allee species because too few dispersers would fail to exceed an Allee threshold resulting in no invasion (Ackleh et al., 2007). In application, movement of invasive species is often reduced through regulations and inspection stations. For example, the United States Department of Agriculture restricts the movement of firewood to reduce accidental transport of forest pests such as the emerald ash borer, and to restrict spread beyond the range borders (Tobin, Diss-Torrance, Blackburn, \& Brown, 2010).

The most interesting aspects of Allee effects occur in low density populations where growth is too small to support these populations and, thus, the populations tend to die out. 
Thus, management strategies that disproportionately reduce low density populations will tend to alter an Allee threshold, $A$. One such strategy is mating disruption, where reductions in mate-finding ability can result in or enhance Allee effects. Mating disruption can be achieved in a number of ways. In insect pests, mating disruption is achieved by saturating the environment with a synthetic pheromone. Although the precise mechanism by which this method reduces mating is unknown, it is suspected that the high concentration of pheromone cues either discourage males from searching for mates, or inhibits the males ability to pinpoint females (Yamanaka \& Liebhold, 2009). Capturing large numbers of males in pheromone-baited traps could also increase Allee effects by removing males from the population (Yamanaka, 2007).

Some management strategies focus on suppressing high density populations. An example of this type of management includes localized pesticide or pathogen applications on infested areas. Recent theoretical work demonstrates how suppressing established populations behind an invasion front can reduce the invasion rate (Walter, Johnson, Tobin, \& Haynes, 2015). Manual removal of a pest or its resource in infested areas may also fall in this category (Johnson, Moran, \& Driml, 1990). These types of strategies would disproportionately reduce high density populations, thus, would alter the effective carrying capacity in patch $1, K_{1}$.

To analyse the model we note the following. The Jacobian of the system $(2)$ is: $J(F(x, y))$ $=\left[\begin{array}{ll}j_{11} & j_{12} \\ j_{21} & j_{22}\end{array}\right]$ where

$$
\begin{aligned}
& j_{11}=-1 / 2 \mathrm{e}^{-\frac{r_{1}\left(K_{1}-x\right)\left(-x+A_{1}\right)}{K_{1}(C+x)}}\left(A_{1} C d r_{1} x+A_{1} K_{1} d r_{1} x+C K_{1} d r_{1} x\right. \\
& -2 C d r_{1} x^{2}-d r_{1} x^{3}-2 A_{1} C r_{1} x-2 A_{1} K_{1} r_{1} x+C^{2} K_{1} d+2 C K_{1} d x \\
& -2 C K_{1} r_{1} x+4 C r_{1} x^{2}+K_{1} d x^{2}+2 r_{1} x^{3}-2 C^{2} K_{1}-4 C K_{1} x \\
& \left.-2 K_{1} x^{2}\right) K_{1}^{-1}(C+x)^{-2} \\
& j_{12}=1 / 2 d \mathrm{e}^{-\frac{r_{2}\left(K_{2}-y\right)\left(-y+A_{2}\right)}{K_{2}(C+y)}}\left(A_{2} C r_{2} y+A_{2} K_{2} r_{2} y+C K_{2} r_{2} y-2 C r_{2} y^{2}\right. \\
& \left.-r_{2} y^{3}+C^{2} K_{2}+2 C K_{2} y+K_{2} y^{2}\right) K_{2}^{-1}(C+y)^{-2} \\
& j_{21}=1 / 2 \mathrm{de} \mathrm{e}^{-\frac{r_{1}\left(K_{1}-x\right)\left(-x+A_{1}\right)}{K_{1}(C+x)}}\left(A_{1} C r_{1} x+A_{1} K_{1} r_{1} x+C K_{1} r_{1} x-2 C r_{1} x^{2}\right. \\
& \left.-r_{1} x^{3}+C^{2} K_{1}+2 C K_{1} x+K_{1} x^{2}\right) K_{1}^{-1}(C+x)^{-2} \\
& j_{22}=-1 / 2 \mathrm{e}^{-\frac{r_{2}\left(K_{2}-y\right)\left(-y+A_{2}\right)}{K_{2}(C+y)}}\left(A_{2} C d r_{2} y+A_{2} K_{2} d r_{2} y+C K_{2} d r_{2} y\right. \\
& -2 C d r_{2} y^{2}-d r_{2} y^{3}-2 A_{2} C r_{2} y-2 A_{2} K_{2} r_{2} y+C^{2} K_{2} d+2 C K_{2} d y \\
& -2 C K_{2} r_{2} y+4 C r_{2} y^{2}+K_{2} d y^{2}+2 r_{2} y^{3}-2 C^{2} K_{2}-4 C K_{2} y \\
& \left.-2 K_{2} y^{2}\right) K_{2}^{-1}(C+y)^{-2}
\end{aligned}
$$

Let $\operatorname{tr}(J(x, y))$ and $\operatorname{det}(J(x, y))$ be the trace and the determinant of the Jacobian evaluated at $(x, y)$, respectively. We will use the Jury condition for quadratic equations to determine the stability of equilibria of the system (2). 


\section{Results}

Here, we justify the use of this model by proving the expanded two-patch model has the properties of a species with a strong Allee effect. Since Allee species have depressed population growth at low population densities, it is then important for the patch model to also have this property. Clearly the origin is an equilibrium, since $F(0,0)=(0,0)$. Thus, the extinction state is an equilibrium. We now determine the local stability of the extinction state.

We first show the system is well defined.

Proposition 1: For system 2, where $F: \Re_{+}^{2} \rightarrow \Re_{+}^{2}$ orbits are bounded.

Proof: Let $\left(x_{1}, x_{2}\right) \in \mathfrak{R}_{+}^{2}$. Define $G\left(x_{1}, x_{2}\right)=F_{1}\left(x_{1}, x_{2}\right)+F_{2}\left(x_{1}, x_{2}\right)$, so

$$
\begin{aligned}
G\left(x_{1}, x_{2}\right)= & x_{1}(t) \exp \left(r_{1}\left[1-\frac{x_{1}(t)}{K_{1}}\right]\left[1-\frac{A_{1}+C}{x_{1}(t)+C}\right]\right) \\
& +x_{2}(t) \exp \left(r_{2}\left[1-\frac{x_{2}(t)}{K_{2}}\right]\left[1-\frac{A_{2}+C}{x_{2}(t)+C}\right]\right) .
\end{aligned}
$$

Now note that

$$
G\left(x_{1}, x_{2}\right)<x_{1}(t) \exp \left(r_{1}\left[1-\frac{x_{1}(t)}{K_{1}}\right]\right)+x_{2}(t) \exp \left(r_{2}\left[1-\frac{x_{2}(t)}{K_{2}}\right]\right) .
$$

Further letting $r^{*}=\max \left\{r_{1}, r_{2}\right\}$ and $K^{*}=\max \left\{K_{1}, K_{2}\right\}$, then

$$
G\left(x_{1}, x_{2}\right)<x_{1} \exp \left(r^{*}\left[1-\frac{x_{1}}{K *}\right]\right)+x_{2} \exp \left(r^{*}\left[1-\frac{x_{2}}{K *}\right]\right) .
$$

Define the map $H: \Re_{+} \rightarrow \Re_{+}$to be $H(x)=x \exp \left(r^{*}\left[1-\frac{x}{K^{*}}\right]\right)$. Thus, we have $G\left(x_{1}, x_{2}\right)<H\left(x_{1}\right)+H\left(x_{2}\right)$. For $x>K^{*}, H(x)<x$ and so iteration of $H$ applied to $x$ must enter or limit on the interval $\left[0, K^{*}\right]$. Thus, orbits of $H$ are bounded by the set $H\left(\left[0, K^{*}\right]\right)$. Since the orbits of $H$ are bounded, the orbits of $G$ are bounded and hence, the orbits of $F$ must also be bounded. This completes the proof.

\subsection{Stability of the extinction state}

The origin, $(0,0)$, is the extinction state. If a strong Allee effect is present, then the origin should be locally attracting. We show this is true under any realistic combination of the parameter values.

Theorem 1: The origin is locally stable.

Proof: To show the origin is locally attracting we use the Jury condition and show $|\operatorname{tr}(J(0,0))|<1+\operatorname{det}(J(0,0))<2$, where

$$
\begin{aligned}
\operatorname{tr}(J(0,0)) & =\left(1-\frac{d}{2}\right)\left(e^{r_{1}\left(1-\frac{A_{1}+C}{C}\right)}+e^{r_{2}\left(1-\frac{A_{2}+C}{C}\right)}\right), \text { and } \\
\operatorname{det}(J(0,0)) & =(1-d) e^{r_{1}\left(1-\frac{A_{1}+C}{C}\right)} e^{r_{2}\left(1-\frac{A_{2}+C}{C}\right)} .
\end{aligned}
$$


Let $\alpha=\exp \left\{r_{1}\left(1-\frac{A_{1}+C}{C}\right)\right\}$ and $\beta=\exp \left\{r_{2}\left(1-\frac{A_{2}+C}{C}\right)\right\}$, then

$$
\operatorname{tr}(J(0,0))=\left(1-\frac{d}{2}\right)(\alpha+\beta) \text { and } \operatorname{det}(J(0,0))=(1-d) \alpha \beta
$$

Note that $|\operatorname{tr}(J(0,0))|<1+\operatorname{det}(J(0,0))$ is equivalent to $-(1+\operatorname{det}(J(0,0)))<\operatorname{tr}(J(0,0))<$ $1+\operatorname{det}(J(0,0))$. Since $0<\alpha, \beta<1$ and $d \in[0,1], \operatorname{tr}(J(0,0))>0$ and $\operatorname{det}(J(0,0)) \geq 0$. So, clearly, $-(1+\operatorname{det}(J(0,0)))<\operatorname{tr}(J(0,0))$.

Next rewriting $\operatorname{tr}(J(0,0))<1+\operatorname{det}(J(0,0))$ using $\alpha$ and $\beta$ gives

$$
\left(1-\frac{d}{2}\right)(\alpha+\beta)<1+(1-d) \alpha \beta .
$$

Rearranging terms this becomes

$$
(\alpha+\beta)-d\left(\frac{\alpha+\beta}{2}\right)<(1+\alpha \beta)-d \alpha \beta
$$

This inequality will hold if we have $\alpha+\beta<1+\alpha \beta$ and $d\left(\frac{\alpha+\beta}{2}\right) \geq d \alpha \beta$. We claim that these last two inequalities hold. For the first inequality, $\alpha+\beta<1+\alpha \beta$ holds if and only if $(\alpha+\beta)-\alpha \beta<1$. This is true if and only if $(\alpha-\alpha \beta)+\beta<1$, which is true if and only if $\alpha(1-\beta)<1-\beta$. But this is true since $\alpha<1$.

For the second inequality, if $d=0$, then $d\left(\frac{\alpha+\beta}{2}\right) \geq d \alpha \beta$ automatically is true. On the other hand, if $d>0$, then since $0<\alpha, \beta<1, \alpha+\beta>2 \alpha \beta$. This implies that $\frac{\alpha+\beta}{2}>\alpha \beta$, and multiplying by $d>0$, gives the inequality $d\left(\frac{\alpha+\beta}{2}\right)>d \alpha \beta$. Thus, we have $d\left(\frac{\alpha+\beta}{2}\right) \geq d \alpha \beta$. This completes the proof.

\subsection{Stability of the threshold equilibrium}

For the next part of the analysis we confirm that the two-patch model has an Allee threshold. To do this we consider the two patches having essentially identical environments. In general, for many situations where the patches are nearby similar patch environments will exist. To do this let $r=r_{1}=r_{2}, A=A_{1}=A_{2}$ and $K=K_{1}=K_{2}$. Under these conditions it is easy to see that $(A, A)$ and $(K, K)$ are also equilibria.

First consider the threshold equilibrium $(A, A)$. The Jacobian evaluated at this population density is:

$$
J(F(A, A))=\left[\begin{array}{cc}
\left(1-\frac{d}{2}\right)\left(1+\frac{A r\left(1-\frac{A}{K}\right)}{C+A}\right) & \frac{d}{2}\left(1+\frac{\operatorname{Ar}\left(1-\frac{A}{K}\right)}{C+A}\right) \\
\frac{d}{2}\left(1+\frac{\operatorname{Ar}\left(1-\frac{A}{K}\right)}{C+A}\right) & \left(1-\frac{d}{2}\right)\left(1+\frac{\operatorname{Ar}\left(1-\frac{A}{K}\right)}{C+A}\right)
\end{array}\right],
$$

which gives 


$$
\begin{aligned}
\operatorname{tr}(J(A, A)) & =\frac{(d-2)\left(r A^{2}-A K r-K A-C K\right)}{K(C+A)}, \text { and } \\
\operatorname{det}(J(A, A)) & =-\frac{\left(r A^{2}-A K r-K A-C K\right)^{2}(d-1)}{K^{2}(C+A)^{2}} .
\end{aligned}
$$

Letting $\alpha=1-d$ and $\beta=\frac{\operatorname{Ar}\left(\frac{A}{K}-1\right)}{A+C}-1$, we rewrite the above to be

$$
\begin{aligned}
\operatorname{tr}(J(A, A)) & =-(\alpha+1) \beta, \\
\operatorname{det}(J(A, A)) & =\alpha \beta^{2} .
\end{aligned}
$$

Using this we show that this equilibrium is unstable.

Theorem 2: The threshold equilibrium, $(A, A)$, is unstable for patches with similar environments.

Proof: Note that since $A<K$, we have that $\beta<-1$. To show that $(A, A)$ is unstable, one of the following conditions must be true:

$$
\begin{aligned}
-(\alpha+1) \beta & >1+\alpha \beta^{2}, \\
-(\alpha+1) \beta & <-1-\alpha \beta^{2}, \text { or } \\
\alpha \beta^{2} & >1 .
\end{aligned}
$$

If the first condition holds then:

$$
\begin{aligned}
-(\alpha+1) \beta & >1+\alpha \beta^{2}, \\
0 & >1+(\alpha+1) \beta+\alpha \beta^{2}
\end{aligned}
$$

If we assume equality, then the roots of $\beta$ in terms of $\alpha$ are $\beta=-\frac{1}{\alpha},-1$. Since $0<\alpha<1$ we must have $-\frac{1}{\alpha}<\beta<-1$ in order for the inequality to be satisfied.

Recall that we have that $\beta<-1$. Now, either $\beta>-\frac{1}{\alpha}$ or not. If not, then $\beta \leq-\frac{1}{\alpha}$, which implies that $\alpha \beta \leq-1$ and since $\beta<-1$ we get $\alpha \beta^{2}>1$. Thus, the third condition is met and $(A, A)$ is unstable. Thus $(A, A)$ is unstable.

\subsection{Stability of the carrying capacity}

As the final step in justifying the model we next consider the equilibrium at the carrying capacity, $(K, K)$. We have:

$$
J(F(K, K))=\left[\begin{array}{cc}
\left(1-\frac{d}{2}\right)\left(1-r\left(1-\frac{C+A}{C+K}\right)\right) & \frac{d}{2}\left(1-r\left(1-\frac{C+A}{C+K}\right)\right) \\
\frac{d}{2}\left(1+r\left(1-\frac{C+A}{C+K}\right)\right) & \left(1-\frac{d}{2}\right)\left(1-r\left(1-\frac{C+A}{C+K}\right)\right)
\end{array}\right]
$$

and 


$$
\begin{aligned}
\operatorname{tr}(J(K, K)) & =-\frac{(d-2)(r A-r K+C+K)}{C+K}, \text { and } \\
\operatorname{det}(J(K, K)) & =-\frac{(r A-r K+C+K)^{2}(d-1)}{(C+K)^{2}}
\end{aligned}
$$

Rewriting this gives,

$$
\begin{aligned}
\operatorname{tr}(J(K, K)) & =(2-d)\left(\frac{r(A-K)}{C+K}+1\right), \text { and } \\
\operatorname{det}(J(K, K)) & =(1-d)\left(\frac{r(A-K)}{C+K}+1\right)^{2} .
\end{aligned}
$$

Theorem 3: Under similar environments the carrying capacity equilibrium, $(K, K)$, is locally stable if:

$$
\frac{r(A-K)}{C+K}+1<\frac{1}{\sqrt{1-d}} .
$$

Proof: Let $\gamma=\left(\frac{r(A-K)}{C+K}+1\right)$. To show stability from the Jury condition we need to show $(2-d) \gamma<(1-d) \gamma^{2}+1<2$. Next rewriting the first part of the inequality we have $0<-(2-d) \gamma+(1-d) \gamma^{2}+1$. Making this an equality and solving for $\gamma$ gives

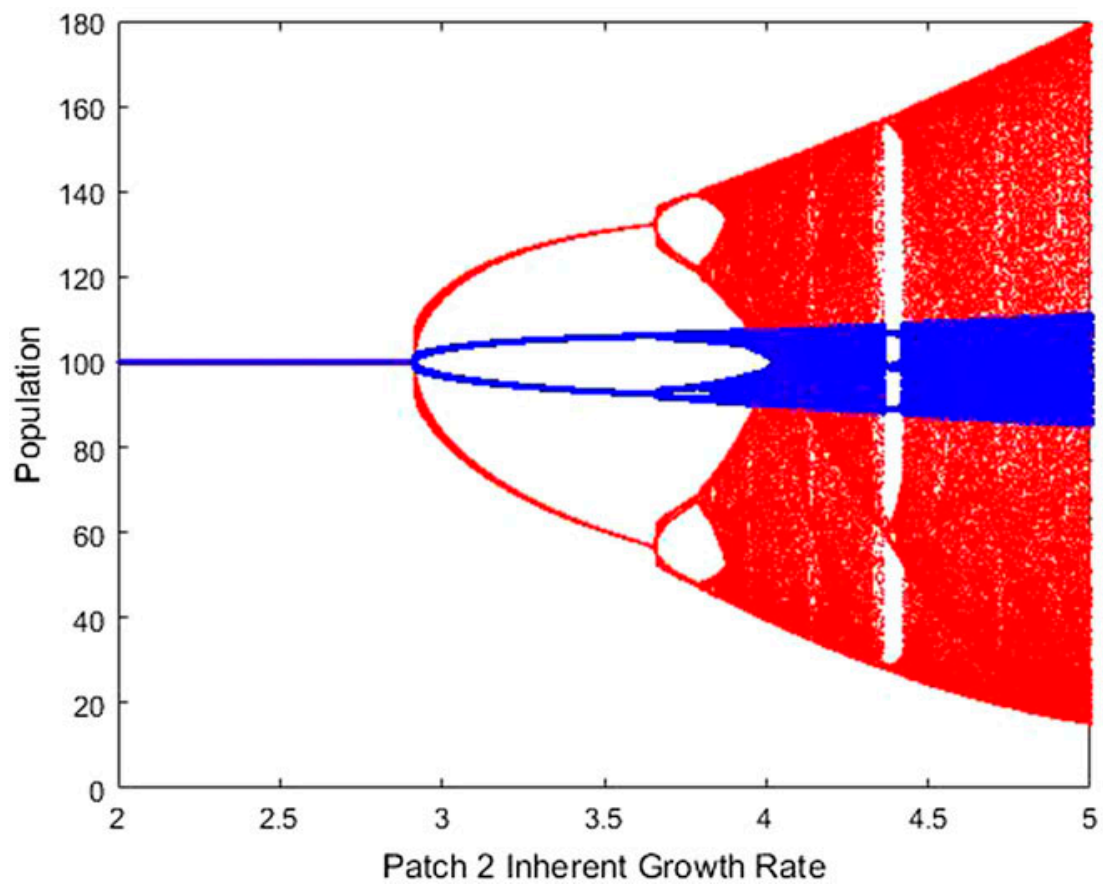

Figure 1. For $d=.2, r_{1}=2, K_{1}=K_{2}=100, A_{1}=A_{2}=20$, and $C=10, r_{2}$ is varied in 2 to produce the bifurcation diagram.

Note: A period doubling bifurcation is observed where the populations in patches 1 and 2 are denoted by the blue and the red, respectively. 
Table 1. Parameter values.

\begin{tabular}{lc}
\hline Parameter & Value \\
\hline$d$ & .5 \\
K & 100 \\
$r$ & 1.5 \\
A & 20 \\
C & 10 \\
\hline
\end{tabular}

$\gamma=1, \frac{1}{1-d}$. Since $0 \leq d \leq 1,1 \leq \frac{1}{1-d}$. However, note that since $A-K<0$ we have that $\gamma<1$, thus, this inequality is always satisfied.

Now consider the other inequality, $(1-d) \gamma^{2}+1<2$. This implies that $(1-d) \gamma^{2}<1$ and that $\gamma<\frac{1}{\sqrt{1-d}}$, which is true by assumption. Thus, $(K, K)$ is locally stable.

In fact this equilibrium loses stability and goes through a period-doubling bifurcation (see Figure 1). Here, the inherent growth rate in the second patch, $r_{2}$, is varied and the resulting populations in both patches are plotted. One can see that the period doubling occurs in both patches though the spread in the oscillations, which are more pronounced in patch 2 (red), and less in patch 1 (blue) where the inherent growth rate is fixed.

\subsection{Simulations}

Management strategies for invasive species are generally not applied uniformly across a landscape for logistic, economic, and/or ecological reasons. For example, management that disproportionately affects high or low density populations are most effectively applied to the established and non-established range of an invading species, respectively. Due to the non-linearity of this model when the environments in the two patches are not similar, stability analyses are not possible. Instead, to examine the effect of management strategies where the environments are purposely changed, we use numerical simulations to predict the outcomes of these strategies.

Starting with the values in Table 1 , we vary different parameters to examine the variations in the asymptotic behaviour of the model. In particular we assume initially the population in patch 1 is at carrying capacity and there is no population in patch 2 , then we look under what conditions the species is able to successfully invade into patch 2 .

We first consider the individual effects of parameter values on invasion success, and then explore how changes in multiple parameter combinations affect invasion success. In particular, we are interested in the efficacy of altering parameters in populations with low, medium and high intrinsic growth rates. In the following results, the model is iterated for 1800 generations, then the next 200 generations are plotted resulting in the following graphs. The populations in both patches are shown.

\subsubsection{Dispersal}

First we examine the effect of varying the diffusion rate, $d$. As expected, increased diffusion allows for greater proportions of dispersers that in turn helps to facilitate invasion. In Figure 2(A), we see that when inherent growth rate is small, reducing the diffusion rate to less than $5 \%$ prevents invasion. At higher values of the diffusion rate, patch 2 is invaded and the carrying capacity is reached in both patches. If the inherent growth rate is increased 

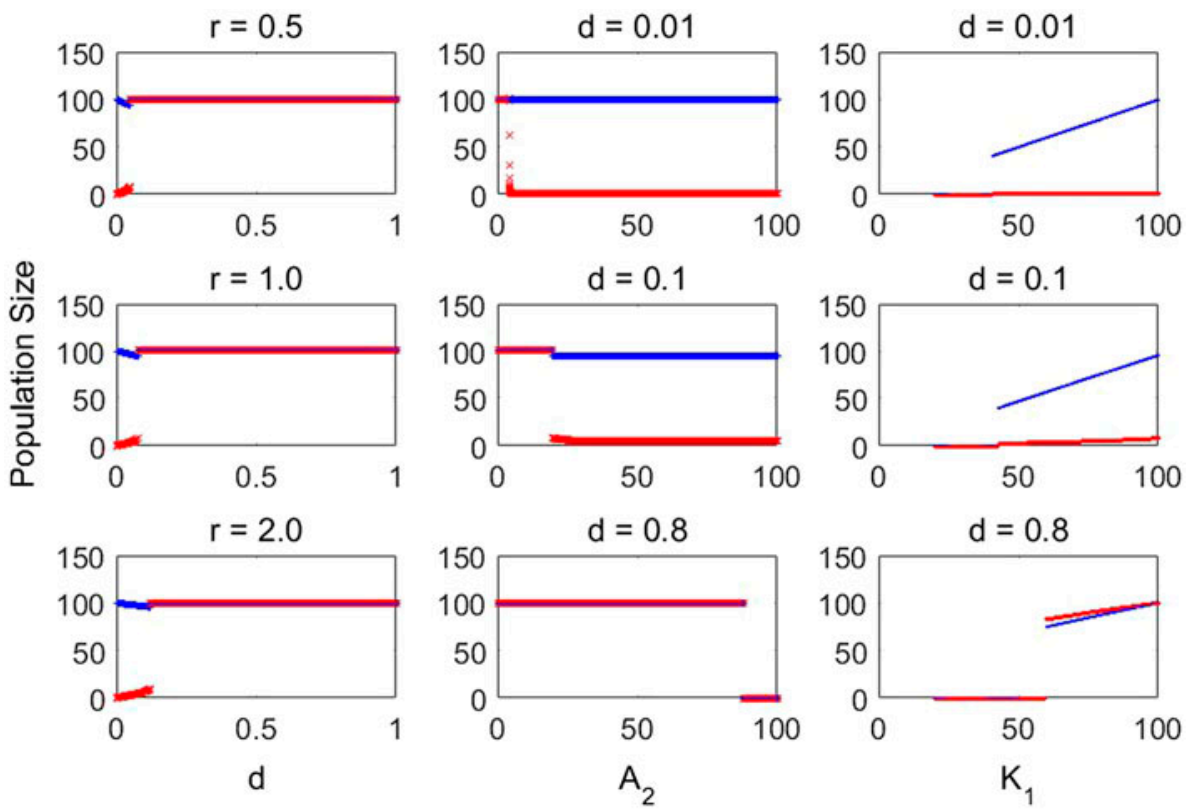

Figure 2. Parameter values used are $d=.2, r_{1}=r_{2}=2, K_{1}=K_{2}=100, A_{1}=A_{2}=20$ and $C=10$ except where noted and varied.

Notes: Bifurcation plots of two-patch models connected through dispersal with initial conditions of patch 1 (blue) population size $(N)$ equal to carrying capacity $(K)$ and patch 2 (red) unoccupied $(N=0)$. Plots A-C show non-transient dynamics across a range of dispersal proportions at $r=.5,1.0$, and 2.0. Plots D-F show non-transient dynamics across a range of Allee thresholds $(A)$ in patch 2 at dispersal proportion $d=.01, .1$ and .8 . Plots $\mathrm{G}-\mathrm{I}$ show non-transient dynamics across a range of carrying capacities in patch 1 at dispersal proportion $d=.01, .1$ and .8 .

from $r=1-1.5$ we see a similar situation in Figure 2(B) and (C). Here, the diffusion percentage necessary to allow expansion is about 7 and $10 \%$, respectively. A higher diffusion rate is required for invasion in models with higher growth rates. This paradoxical result is due to the fact that increases in $r$ also increases the strength of the Allee effect (Figure 3 ).

Below the respective dispersal thresholds, the non-transient state is a source-sink dynamic. Here, the density of the source population in patch 1 is reduced to below carrying capacity by a net emigration to patch 2 , and patch 2 is maintained at a non-zero density by a net immigration (Holt, 1983).

\subsubsection{Allee threshold}

The Allee threshold in patch 2 is varied from 0 to the carrying capacity to explore the effect of different thresholds under simulated elevation of the Allee threshold at the invasion front. We see in Figure 2(D) and (E) that when diffusion rates are low, a low Allee threshold (about 5 and 20, respectively) is sufficient to prevent invasion from patch 1 into patch 2 . However, as the diffusion percentage increases to .8, see Figure 2(F), a threshold very close to the carrying capacity is required and beyond this threshold both populations go extinct.

Consequently, a higher threshold is required to inhibit invasion with higher diffusion rates. Also with higher diffusion rates comes the possibility of extinction in both patches because the higher movement of dispersers drains the population from the first patch to the point where the population may drop below the Allee threshold. 


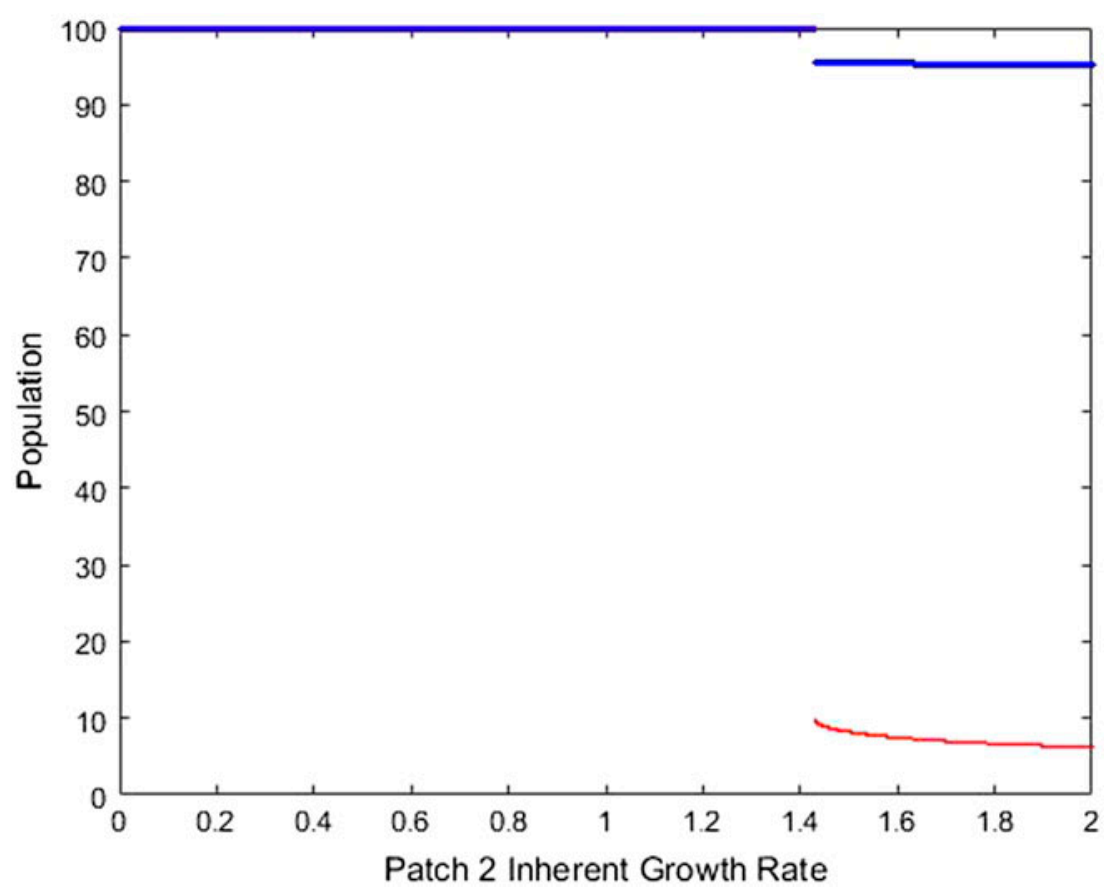

Figure 3. Bifurcation plot of a two-patch model connected through dispersal with initial conditions of patch 1 (blue) population size $(N)$ equal to carrying capacity $(K)$ and patch $2($ red) unoccupied $(N=0)$. Notes: Parameter values used are $d=.1, r_{1}=r_{2}=2, K_{1}=K_{2}=100, A_{1}=A_{2}=20$ and $C=10$. On the horizontal axis we vary the inherent growth rate in the second patch.

\subsubsection{Carrying capacity}

The carrying capacity in patch 1 was varied from the Allee threshold to the carrying capacity of patch 2 to simulate suppression of high density populations in the established range. At low diffusion rates, there is no invasion because with a lower carrying capacity very few dispersers move into patch 2, see Figure $2(\mathrm{G})$ and $(\mathrm{H})$. In fact, a low carrying capacity in patch 1 will cause extinction in that patch, even at a low diffusion rate because emigration reduces the population size to below the Allee threshold.

At higher diffusion rates, see Figure 2(I), large carrying capacities will facilitate successful invasion of patch 2 because the high equilibrium densities in patch 1 donate a high number of dispersers to patch 2 . This is required to allow for both patch populations to exceed and remain above the Allee threshold.

\subsubsection{Population growth rate}

Changing the inherent growth rate in patch 2 affects the ability of a population to invade in ways that seem initially counterintuitive. Increasing the inherent growth rate increases the diffusion rate necessary for invasion into patch 2 . This increase in the diffusion rate can be seen in Figure 2(A), (B), and (C). Relatedly, reducing $r_{2}$ reveals that populations with low growth rates are more likely to invade than those with high growth rates. At low diffusion and very low values of growth rate, see Figure 3, the population expands into the second patch, but at the higher growth rates invasion is unsuccessful. 


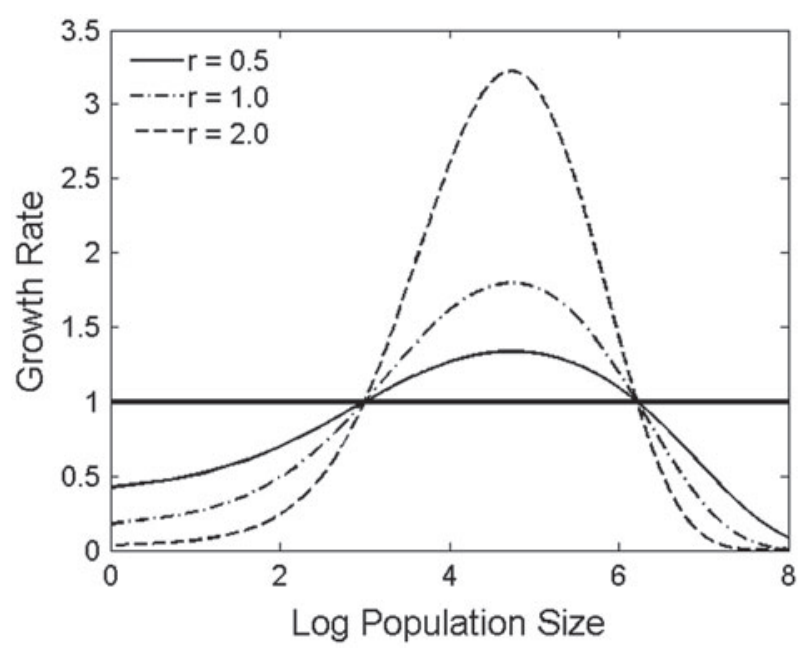

Figure 4. Density-dependent population growth rates using Equation (1) with the following parameters: $K=500, A=20, C=0, r=.5,1.0$ and 2.0.

Notes: This demonstrates that populations with a higher per capita growth rate $(r)$ have greater depression in growth rate below the Allee threshold.

The reason for this unexpected result is explained from the model. When populations are small, the exponent of the growth rate is negative due to the factor $\left(1-\frac{A_{2}+C}{x_{2}(t)+C}\right)$. Increasing $r$ results in more negative growth rates in the low density populations, which restricts invasion. In effect the larger inherent growth rates cause a stronger Allee effect, which require greater dispersal for successful invasion. This is visually illustrated in Figure 4 where the rate of depression in growth rate is most depressed below the Allee threshold when per capita growth rate is high.

\subsection{Context-dependent invasion management}

Invasive species can be managed using one or a combination of alternative strategies, each of which disproportionately affects certain population parameters more than others. The efficacy of different management methods for preventing invasion can be contextdependent, i.e. may differ for populations with low vs. high Allee effects, or those in highly isolated vs. highly connected populations. This may result in optimal management strategies that are species-specific, or that differ geographically within a single species.

Here, we use the gypsy moth as an example to illustrate how our results may be used to assess the effectiveness of different invasion management strategies. While, the population estimates we use here are empirically-based, they have varying degrees of confidence, e.g. some have large uncertainty and are generalized over large scales. Still, these results are a useful starting point for elucidating the effectiveness of different management strategies for gypsy moth invasion.

\subsubsection{Gypsy moth - example}

The European gypsy moth (Lymantria dispar) is a non-native forest defoliator in North America. The gypsy moth was introduced near Boston, MA in 1869, and has since spread 
North into Southern Canada, South through Virginia and into North Carolina, and West into Wisconsin and Northern Minnesota (Tobin, Whitmire, Johnson, Bjornstad, \& Liebhold, 2007). The gypsy moth is a generalist feeder, but performs best on host trees in the genera Quercus (oaks), Populus (poplar), and Larix (larch) (Morin et al., 2005). Most years the gypsy moth is at low densities, but displays periodic outbreaks where large tracts of forest are defoliated (Johnson, Liebhold, \& Bjornstad, 2006a).

Since 1996, the Slow the Spread Program has deployed on the order of 100,000 pheromone-baited traps to monitor the gypsy moth invasion front and reduce range expansion (Tobin, Bai, Eggen, \& Leonard, 2012). The traps serve a dual purpose: they provide a detailed record of gypsy moth invasion, and facilitate detection of nascent populations growing beyond the invasion front. The USDA focuses eradication efforts on these nascent populations to reduce spread. The programme has been declared successful at reducing the spread of the gypsy moth from an annual rate of approximately $20 \mathrm{~km} / \mathrm{yr}$ down to $10 \mathrm{~km} / \mathrm{yr}$ (Tobin et al., 2004). Analysis of the pheromone-baited trap catches in low density populations at the invasion front reveals strong evidence for Allee effects in some geographic regions along the gypsy moth invasion front (Johnson et al., 2006a; Tobin et al., 2007).

A number of strategies are used to manage gypsy moth populations. Pheromone-baited traps may be effective at disrupting mate-finding in low density gypsy moth populations by reducing population growth rates and potentially magnifying Allee effects. Pheromonebaited traps are not effective at reducing growth rates in high density populations, which are often found in the established range of the gypsy moth. Suppressing high density populations is more effective through focused application of either pesticides or a natural enemy such as the Bacillus thuringiensis virus. While, this method is primarily used to reduce negative impacts on local forests, it can have a secondary effect of reducing the number of dispersers moving from the established range to beyond the invasion front, which is thought to be a driver of gypsy moth range expansion (Walter et al., 2015). Finally, the number of dispersers can also be reduced by restricting the anthropogenic movement of gypsy moth life stages, such as egg masses laid on timber products, through a combination of governmental restrictions, public information campaigns and monitoring stations. The most effective strategy or combination of strategies may be dependent on the particulars of the underlying population properties (Tobin et al., 2011).

Spatio-temporal population dynamics of the gypsy moth varies geographically (Johnson et al., 2006a). In western Virginia and West Virginia, gypsy moth outbreaks occur periodically and the spread rate has been approximately $10 \mathrm{~km} / \mathrm{yr}$ over the last couple of decades. In contrast, Wisconsin has never experienced densities sufficient to cause defoliation, but the spread rate commonly exceeds $20 \mathrm{~km} / \mathrm{yr}$. The invasion front is approximately static and outbreaks are rare in the mid-western states of Ohio, Indiana and Illinois (Tobin et al., 2007), an agriculturally intense region with a low abundance of preferred hosts (Morin et al., 2005).

Geographic variation in spread rates across regions seems to be correlated with variation in local population processes. In Virginia and West Virginia, the Allee threshold is at approximately 20 males per trap. In contrast, the Allee threshold in Wisconsin is only approximately 1-2 males per trap (Tobin et al., 2007). Winter egg mortality is likely greater in Wisconsin due to minimum temperatures dropping below egg survival thresholds (Campbell, 1973); thus, likely resulting in a reduction in the density-independent growth 


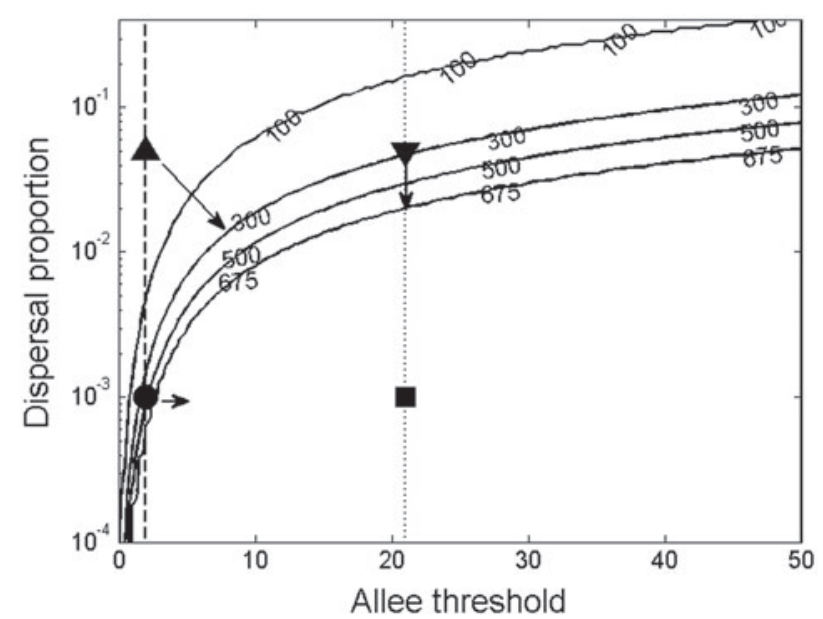

Figure 5. Invasion potential plot based on gypsy moth population parameters.

Notes: The contour plot represents the carrying capacity that the population in patch 1 must exceed to contribute enough dispersers to invade patch 2. Thus, the figure space to the lower right of the 675 contour line would represent no invasion in populations with carrying capacities above $K=675$. In contrast, the figure space to the upper left of 100 contour line would be invaded by populations with carrying capacities above $K=100$. The Allee threshold in patch 2 and the dispersal proportion of the system are plotted on the $x$ - and $y$-axes, respectively. Other model parameters are per capita growth rate $r=2.7$ and Allee shape parameter $C=10$. Vertical dashed line indicates Allee threshold in Wisconsin and vertical dotted line indicates estimated Allee threshold for West Virginia and Western Virginia (Tobin et al., 2007). The Allee threshold is not well defined for Illinois-Ohio. Lower dispersal proportions would be expected in more fragmented habitats with greater isolation. The triangle and inverted triangle indicate areas with high dispersal, as may be found in contiguous forest, in areas with low and high Allee thresholds, respectively. The circle and square indicate populations with low dispersal in highly fragmented habitats with low and high Allee thresholds, respectively. Arrows indicate optimal suppression of dispersal and/or inflation of Allee effects through management strategies.

rate in the gypsy moth. Dispersal rates in the mid-western states of Illinois, Indiana and Ohio are likely reduced due to a low abundance of preferred host trees.

We created an invasion contour plot based on an empirically estimated per capita growth rate of $r=2.7$ for the gypsy moth (Johnson et al., 2006b), see Figure 5. The four symbols (circle, triangle, inverted triangle and square) represent four general conditions that a gypsy moth population may experience in different geographic locations along the invasion front. The arrows represent the most effective management results to prevent invasion. The triangle represents a population with a low Allee threshold $(A=2)$ in a region with a moderate to high abundance of preferred host trees, thus, with high dispersal. This may be a reasonable characterization of the gypsy moth invasion front in Wisconsin. While, the intrinsic rate of growth in Wisconsin arguably may be depressed due to high winter mortality, comparison of the plots (not shown) reveals that the shape of the contour plots are qualitatively similar and, thus, this detail is unimportant for our purposes.

The inverted triangle is a reasonable representation of a population in West Virginia, where the Allee threshold is high and the forest has a moderate to high abundance of preferred host trees. The circle and square represent gypsy moth populations with low and high Allee thresholds, respectively, from forests with a low abundance of preferred host trees, which tend to be more isolated; thus, where dispersal is low, as in the intensively farmed habitat from Illinois to Ohio.

In the population that is characteristic of Wisconsin (triangle), analysis of the contour plot suggests that using a management strategy that both reduces dispersal and inflates the 
Allee threshold may be most effective. While, pheromone-baited traps are already used to monitor gypsy moth spread along the invasion front, increasing the density of trap arrays in high quality habitat, which are characterized by large basal area of preferred hosts (Liebhold, Halverson, \& Elmes, 1992), and adjacent to established populations, may effectively inflate the Allee threshold. Restricting the movement of egg masses on timber products through governmental policy, public information campaigns and monitoring could complement the effectiveness of the traps by reducing the number of dispersers. Focused suppression in the established range would likely not be as effective because gypsy moth populations rarely reach high densities in this region.

In the population characteristic of West Virginia (inverted triangle), which has an estimated carrying capacity of $K=675$ (Tobin et al., 2007), the plot suggests that inflating the Allee threshold would have little effect at preventing invasion. In contrast, reducing dispersal proportion moderately could prevent invasion by increasing the necessary carrying capacity to above the observed $K=675$ for this region. Thus, this would suggest that governmental regulations that restrict movement of gypsy moth life stages is the most effective strategy for reducing the spread of gypsy moth in West Virginia and the western portion of Virginia. Focused suppression of high density populations through spraying in the established range may also reduce the number of dispersers.

The results suggest that invasion is unlikely in the highly fragmented habitats from Illinois to Ohio (square) when gypsy moth populations have high Allee thresholds, even in the absence of management. This is consistent with the last 20 years during which the gypsy moth invasion front has remained nearly static. However, if conditions were to change such that the Allee threshold decreased (circle), i.e. if a generalist natural enemy were to decline in abundance, then focusing solely on inflating the Allee threshold by increasing trap density is predicted to be the best strategy. This may be feasible because preferred hosts in this region are generally distributed into manageable patches that are small and sparse.

\section{Discussion}

Understanding how Allee effects govern population behaviour is important both for the conservation of rare species and the management of invading populations (Taylor \& Hastings, 2005). Recent research has revealed that Allee effects can cause a rich diversity of patterns in temporal and spatial population dynamics (Clerc, Escaff, \& Kenkre, 2010). Jonsen, Bourchier, and Roland (2007) found that weed control agents with Allee effects had a reduced probability of persistence, particularly when dispersal and stochasticity were increased.

Amarasekare (1998a) used a Levins model modified for metapopulation-level Allee effects, where some fraction of the habitats are occupied to explore metapopulation-level Allee effects. The study focused on how the Allee effect reduced colonization rate at low occupancy levels under increasing habitat destruction. A similar dynamic may emerge from our model if it were extended to beyond two patches, but that is beyond the scope of this study.

Amarasekare also looked at a similar two-patch model, see (Amarasekare, 1998b). In this paper, the author looked at a differential equation model that looked at both negative density dependence and the Allee effect. In particular for the model with Allee effect with 
dispersal, it was seen that populations can survive as a source-sink system. The survival was often at intermediate densities. Here, we investigated invasion instead and saw some survival at intermediate densities as well as full invasion to the carrying capacity in both patches.

In this study, we find that Allee effects could result in source-sink dynamics, consistent with previous work that predicts stable source-sink dynamics at a species range border (Holt, 1983). When Allee effects were high, under certain conditions, such as increased dispersal, global extinction could result. Kang and Armbruster (2011) found a similar pattern of destabilization from source-sink dynamics to global extinction under increased dispersal in a discrete two-patch model that examined plant-insect interactions. In an expanding population with stochasticity, increasing the magnitude of an Allee threshold increases the expected time to colonization (Potapov \& Rajakaruna, 2013).

Kang and Lanchier (2011) used an ordinary differential equation model with an Allee effect to demonstrate that adding stochasticity introduces metastability to the system, where invasion or global extinction are probabilistic consequences. The finding of Tobin et al. reveal empirical evidence that invasion speed of an expanding Allee species is reduced by strong Allee effects. The Tobin et al. (2011) study used a similar discrete model of Ricker form to create synthetic data,

$$
N_{t}=\exp \left(r\left(1-\frac{N_{t-1}}{K}\right)\left(\frac{N_{t-1}-A}{K}\right)\right)
$$

where $r$ is the growth rate, $K$ is the carrying capacity and $A$ is the Allee threshold. Finally, a recent study shows that spatial variation in Allee effects around a mean tends to facilitate more rapid invasion than a homogeneous mean Allee effect (Walter et al., 2015). Together these studies illustrate the important role Allee effects can play in the colonization, persistence and expansion of invasive species.

The two-patch model in our study provides a simple but eloquent framework for exploring spread in a species invasion. The results expand on previous studies in that we link changes in population parameter values to different management strategies. Further, we analyze the effectiveness of separate and combined management methods at preventing range expansion. We were able to explore and compare the effectiveness of management methods on different landscapes that vary in both host abundance and in strengths of Allee effects across the geographic extent of the gypsy moth invasion front.

We showed under similar environments this model has similar Allee properties as a one patch system, i.e. the existence of an Allee threshold equilibrium. One can see in the study done by Franke and Yakubu (1996) that having multiple patches can change the basic behaviour of the model. In their work, the addition of a second patch used as a safe refuge changed the behaviour of the system that was observed in a single patch model.

As expected, invasion in a two-patch model was facilitated by lower Allee thresholds, greater carrying capacities and greater proportions of dispersers. Invasion in an Allee population requires that the Allee threshold is exceeded by dispersers entering an empty patch, which is the product of carrying capacity, i.e. the non-transient population density in our deterministic model, and density-independent dispersal. Thus, decreasing either carrying capacity or dispersal proportion can suppress invasion. Likewise, an inflated Allee threshold increases the necessary number of dispersers to facilitate invasion. 
We found that in this model the increase in inherent growth rate in the second patch aids in inhibiting expansion into that patch. This is counterintuitive, though by examining the model it is clear why this is the case. Specifically, our model has a positive relationship between per capita growth and the strength of positive density-dependent growth around the Allee threshold (Figure 4). This results in faster population decline below the Allee threshold, which would require greater dispersal to be rescued from extinction. Whether this is indicative of natural populations with variance in per capita growth is not clear. We feel that despite this the model demonstrates reasonable conclusions for the application of different management strategies.

Although beyond the scope of this study, adding stochasticity or density-dependent dispersal to the model could have significant effects on invasion. For example, in a stochastic Allee model with high magnitude population fluctuations, invasion occurred in pulses interspersed within periods of stasis (Johnson et al., 2006b). Stochasticity adds uncertainty to Allee populations, and can increase both the probabilities of extinction of established populations and persistence of low density populations (Roth \& Schreiber, 2014), as well as the invasion rate of empty patches (Potapov \& Rajakaruna, 2013).

The efficacy of management strategies at suppressing invasion depends on both the life history of the species and environmental context (Grice, Clarkson, \& Calvert, 2011); thus, understanding the ecology of a species and its environment is critical for effective management. Bogich and Shea (2008) used a metapopulation framework to demonstrate that optimal management strategies can vary geographically in the gypsy moth based on patch sizes and colonization rates, but did not consider Allee effects. Ecologists have recognized that invasive species management can create or enhance Allee effects, although some strategies may be ineffective or even work counter to the benefits of Allee effects (Tobin et al., 2011).

Low abundance of preferred host trees and Allee effects seem to drive the invasion stasis in Illinois, Indiana and Ohio. This region is characterized by small and sparse forest patches of preferred hosts for gypsy moth (Morin et al., 2005). This is consistent with previous research that found gypsy moth with Allee effects have a critical patch size, below which a population cannot persist (Verken, Kramer, Tobin, \& Drake, 2011). This process could restrict expansion of the gypsy moth invasion front through the process of invasion pinning (Keitt et al., 2001). However, some level of forest discontinuity may be beneficial for gypsy moth expansion. Mating success of gypsy moth females was found to be greater at forest edges compared to forest interior (Thompson, Grayson, \& Johnson, 2016). This suggests a unimodal relationship between landscape structure and spread rates. Moreover, it is consistant with the idea that landscape structure can inflate mate-finding Allee effects by reducing dispersal. This relationship is not steadfast, however, because low forest fragmentation is found with high Allee effects in West Virginia.

We used the gypsy moth as an example to illustrate how environmental context can be incorporated into a decision-making framework. Previous research has identified geographic variation in gypsy population dynamics and the underlying demographic parameters including Allee thresholds. Movement restrictions and mating disruptions are two common methods used for managing the spread of invasive species. Given that management resources are limited, it is pragmatic to compare the efficacy of each method to address whether all resources should be devoted to one or the other method, or whether a better strategy is to divide resources between the two methods. We predict 
that movement restrictions through governmental regulations and monitoring are most effective at suppressing invasion in regions of high abundance of preferred hosts and high Allee thresholds, as is found in West Virginia. In contrast, we predict that mating disruption with pheromone-baited traps, which can raise the Allee threshold, is most effective at suppressing invasion in areas with low abundance of preferred hosts, such as in Illinois, Indiana and Ohio. When the abundance of preferred hosts is high but Allee thresholds are low, as in Wisconsin, we predict that dedicating limited resources to both governmental restrictions on movement and enhanced pheromone-baited trap densities would be more effective than either strategy separately.

This study demonstrates that context is important when managing Allee populations, and specifically that different strategies may be ideal for a species in different parts of its geographic range. The framework could be modified to address variation in management efficacy across temporal scales as well, such as when effects differ across a life cycle (Magalhaes \& Walgenbach, 2011). Finally, the generality of the model affords its modification and use for management of a broad range of invasive species.

\section{Acknowledgements}

We would like to thank Jonathan Walter for helpful input on the framework of this manuscript.

\section{Disclosure statement}

No potential conflict of interest was reported by the authors.

\section{Funding}

This research was supported in part by Domestic Cooperative Agreement 13-CA-11420004-231 award by the USDA Forest Service, Northeastern Area, State and Private Forestry to DMJ, and a National Science Foundation, Division of Environmental Biology grant, award number 1556767 to DMJ.

\section{References}

Ackleh, A. S., Allen, L. J., \& Carter, J. (2007). Establishing a beachhead: A stochastic population model with an Allee effect applied to species invasion. Theoretical Population Biology, 71, 290-300.

Amarasekare, P. (1998a). Allee effects in metapopulation dynamics. The American Naturalist, 152, 298-302.

Amarasekare, P. (1998b). Interactions between local dynamics and dispersal: Insights from single species models. Theoretical Population Biology, 53, 44-59.

Anic, V., Henriquez, C. A., Abades, S. R., \& Bustamante, R. (2015). Number of conspecifics and reproduction in the invasive plant eschscholzia Californica (papaveraceae): Is there a pollinatormediated Allee effect? Plant Biology, 17, 720-727.

Bogich, T., \& Shea, K. (2008). A state-dependent model for the optimal management of an invasive metapopulation. Ecological Applications, 18, 748-761.

Campbell, R. (1973). Numerical behavior of a gypsy moth population system. Forest Science, 19, $162-167$.

Chow, Y., \& Jang, S. R.-J. (2014). Allee effects in a ricker-type predator-prey system. Journal of Difference Equations and Applications, 20, 1350-1371. 
Clerc, M. G., Escaff, D., \& Kenkre, V. (2010). Analytical studies of fronts, colonies, and patterns: Combination of the Allee effect and nonlocal competition interactions. Physical Review E, 82. doi:10.1103/PhysRevE.82.036210

Courchamp, F., Ludek, 1., \& Gascoigne, J. (2008). Allee effects in ecology and conservation. New York, NY: Oxford University Press.

Franke, J. E., \& Yakubu, A. (1996). Extinction and persistence of species in discrete competitive systems with a safe refuge. Journal of Mathematical Analysis and Applications, 203, 746-761.

Gregory, S., Bradshaw, C., Brook, B., \& Courchamp, F. (2010). Limited evidence for the demographic Allee effect from numerous species across taxa. Ecology, 91, 2151-2161.

Grice, A., Clarkson, J., \& Calvert, M. (2011). Geographical differentiation of management objectives of invasive species: A case study of Hymenachne amplexicaulis in Australia. Environmental Science \& Policy, 14, 986-997.

Holt, R. (1983). Immigration and the dynamics of peripheral populations. In A. G. J. Rhodin \& K. Miyata (Eds.), Advances in Herpetology and Evolutionary Biology (pp. 680-694). Cambridge: Museum of Comparative Zoology, Harvard University.

Jang, S. (2006). Allee effects in discrete-time host-parasitoid model. Journal of Differential Equations and Applications, 12, 165-181.

Johnson, D., Liebhold, A., \& Bjornstad, O. (2006a). Geographical variation in the periodicity of gypsy moth outbreaks. Ecography, 29, 367-374.

Johnson, D. M., Liebhold, A. M., Tobin, P. C., \& Bjornstad, O. N. (2006b). Allee effects and pulsed invasion by the gypsy moth. Nature, 444, 361-363.

Johnson, D., Moran, P., \& Driml, S. (1990). Evaluation of a crown-of-thorns starfish (acantasterplanci) control program at grub reef. Coral Reefs, 9, 167-171.

Jonsen, I., Bourchier, R., \& Roland, J. (2007). Influence of dispersal, stochasticity, and an Allee effect on the persistence of weed biocontrol introductions. Ecological Modelling, 203, 521-526.

Kang, Y., \& Armbruster, D. (2011). Dispersal effects on a discrete two-patch model for plant-insect interactions. Journal of Theoretical Biology, 268, 84-97.

Kang, Y. \& Lanchier, N. (2011). Expansion or extinction: Deterministic and stochastic two-patch models with Allee effects. Mathematical Biology, 268, 925-973.

Keitt, T., Lewis, M., \& Holt, R. (2001). Allee effects, invasion pinning, and species' borders. American Naturalist, 157, 203-216.

Kramer, A. M., Dennis, B., Liebhold, A. M., \& Drake, J. M. (2009). The evidence for Allee effects. Population Ecology, 51, 341-354.

Lanchier, N. (2013). The role of dispersal in interacting patches subject to an Allee effect. Advances in Applied Probability, 45, 1182-1197.

Liebhold, A., Halverson, J., \& Elmes, G. (1992). Gypsy-moth invasion in North America - A quantitative-analysis. Journal of Biogeography, 19, 513-520.

Li, J., Song, B., \& Wang, X. (2007). An extended discrete ricker population model with Allee effects. Journal of Difference Equations and Applications, 13, 309-321.

Magalhaes, L. C., \& Walgenbach, J. F. (2011). Life stage toxicity and residual activity of insecticides to codling moth and oriental fruit moth. Journal of Economic Entomology, 104, 1950-1959.

Morin, R., Liebhold, A., Luzader, E., Lister, A., Gottschalk, K., \& Twardus, D. (2005). Mapping hostspecies abundance of three major exotic forest pests, USDA Forest Service. Research Paper NE-726, Newtown Square, Pennsylvania.

Potapov, A., \& Rajakaruna, H. (2013). Allee threshold and stochasticity in biological invasions: Colonization time at low propagule pressure. Journal of Theoretical Biology, 337, 1-14.

Ricker, W. (1954). Stock and recruitment. Journal of the Fisheries Research Board of Canada, 11, 559-623.

Roth, G., \& Schreiber, S. J. (2014). Pushed beyond the brink: Allee effects, environmental stochasticity, and extinction. Journal of Biological Dynamics, 8, 187-205.

Suckling, D. M., Tobin, P., McCullough, D. G., \& Herns, D. A. (2012). Combining tactics to exploit Allee effects for eradication of alien insect populations. Journal of Economic Entomology, 105, $1-13$. 
Taylor, C. M., \& Hastings, A. (2005). Allee effects in biological invasions. Ecological Letters, 8, 895-908.

Thompson, L., Grayson, K., \& Johnson, D. (2016). Forest edges enhance mate-finding in the invasive European gypsy moth, lymantria dispar. Entomologia Experimentalis et Applicata, 158, 295-303.

Tobin, P., Bai, B., Eggen, D., \& Leonard, D. (2012). The ecology, and geopolitics, and economics of managing lymantria dispar in the United States. International Journal of Pest Management, 58, 377-377.

Tobin, P. C., Berec, L., \& Liebhold, A. M. (2011). Exploiting Allee effects for managing biological invasions. Ecology Letters, 14, 615-624.

Tobin, P., Diss-Torrance, A., Blackburn, L., \& Brown, B. (2010). What does 'local' firewood buy you? Managing risk of invasive species introduction. Journal of Economic Entomology, 103, 1569-1576. Tobin, P., Sharov, A., Liebhold, A., Leonard, D. S., Roberts, E. A., \& Learn, M. R. (2004). Management of the gypsy moth through a decision algorithm under the sts project. American Entomologist, 50, 200-209.

Tobin, P., Whitmire, S., Johnson, D., Bjornstad, O., \& Liebhold, A. (2007). Invasion speed is affected by geographical variation in the strength of Allee effects. Ecology Letters, 10, 36-43.

Verken, E., Kramer, A., Tobin, P., \& Drake, J. (2011). Critical patch size generated by Allee effect in gypsy moth, lymantria dispar (1.). Ecology Letters, 14, 179-186.

Vitousek, P., D’Antonio, C., Loope, L., \& Westbrooks, R. (1996). Biological invasions as global environmental change. American Scientist, 84, 468-478.

Walter, J., Johnson, D. M., Tobin, P., \& Haynes, K. J. (2015). Population cycles produce periodic range boundary pulses. Ecography, 38, 1200-1211.

Williamson, M., \& Fitter, A. (1996). The varying success of invaders. Ecology, 77, 1661-1666.

Yamanaka, T. (2007). Mating disruption or mass trapping? Numerical simulations analysis of a control strategy for lepidopterna pests. Population Ecology, 49, 75-86.

Yamanaka, T., \& Liebhold, A. (2009). Spatially implicit approaches to understand the manipulation of mating success for insect invasion management. Population Ecology, 51, 427-444. 